\title{
Myocilin Localization in the Canine Eye by Light, Confocal and Electron Microscopy
}

\author{
D.A. Samuelson, H. Tajwar, H. Hart, P.A. Lewis, E.O. MacKay and K.N. Gelatt
}

Department of Small Animal Clinical Sciences, University of Florida, P.O. Box 100126, Gainesville, FL 32610, USA

Primary open-angle glaucoma (POAG) is the second-most common cause of blindness in people in the U.S., resulting in damage to the optic nerve and loss of vision. Similarly, it is a major cause of blindness among many breeds of dogs. Until recently, most information on this disease has been collected on the ways internal pressure within the eye can rise and how optic nerve damage may occur with little information on how POAG begins. Among a handful of genes have been recently discovered to be associated with human POAG, the one most prevalent is called GLC1A, which is involved in the production of a protein called myocilin $[1,2]$. While the roles of myocilin are not entirely clear, it is suggested that it reacts to stressful conditions and may provide protection for the cell and the area outside the cell. Furthermore, evidence is building that defects in GLC1A may be responsible directly for the pathogenesis of POAG. We are presently studying the presence of myocilin in the canine eye with regard to POAG, using a colony of Beagles as an inherited model [3]. In trying to identify the mechanisms responsible for causing this disease in this colony we have begun to test for the presence of myocilin by immunocytochemistry using light microscopy (LM), confocal microscopy (CM) and transmission electron microscopy (TEM). With antibodies that have been made to recognize myocilin, eye specimens have been examined for the presence of myocilin. It is proposed that differences in the amount of myocilin in specific regions of the eye will occur when comparing normal specimens to those with the inherited disease.

Single anterior portions of each eye have been immersed in $10.0 \%$ neutral buffered formalin or 4.0 $\%$ paraformaldehyde for $24 \mathrm{hr}$ for LM, CM and TEM analyses, respectively. For LM and CM, sections were incubated sequentially with washes between incubations, first in peroxidase block (DAKO Inc.), followed by goat serum, for $20 \mathrm{~min}$ each at RT, then with the primary antibody, rabbit polyclonal anti-human MYOC IgG (Santa Cruz Biotechnology ) overnight at $4^{\circ} \mathrm{C}$, followed with secondary antibody, either biotinylated link followed by peroxidase-labeled streptavidin and then by substrate-chromogen AEC for 10 at RT or fluorescein-labeled Texas red for $1 \mathrm{hr}$. Age-matched sections from each group of specimens were compared, and examined for similarities and differences using LM. For TEM, specimens on nickel grids were incubated with bovine serum albumin for $10 \mathrm{~min}$ followed by goat serum for $1 \mathrm{hr}$, both at RT. The specimens were incubated with primary antibody, rabbit polyclonal anti-human MYOC IgG (Santa Cruz Biotechnology), followed by incubation with the secondary antibody, $18 \mathrm{~nm}$ colloidal gold labeled goat anti-rabbit IgG (Jackson Immuno) both at RT for 1 hour. Grids were then examined using TEM.

Negative controls were performed in both procedures by primary antibody exclusion.

Within normal, preglaucomatous and moderately glaucomatous canine specimens from age groups three months to thirteen years of age, identical immunolabeling of myocilin was observed by light microscopy. Immunolabeling in these specimens showed that myocilin was homogeneously distributed in ocular tissues. Immunolabeling of specimens from canines with advanced glaucoma, however, exhibited an increased aggregation in areas where aqueous humor leaves the eye, the iridocorneal angle (ICA), nonpigmented (NP) epithelium of the ciliary processes, and anterior 
cornea. In canine specimens with advanced glaucoma, greater intensity of staining was observed within the area of the ICA, and the NP epithelium of the ciliary processes. Localization by CM and TEM produced results similar to those observed by LM. In addition, both revealed myocilin within apically positioned vesicles in the NP epithelium as well as detection of the label within melanocytes, adhering to the surface of individual melanosomes (Figs. 1-3).

This is the first study in which localization on myocilin in the normal and glaucomatous canine eye was successfully performed by immunocytochemical techniques. The combination of LM, CM, and TEM in this study allowed us to determine the precise localization of protein being studies at both the cellular and tissue level. Immunolocalization by TEM enabled us to evaluate pigmented cells that are difficult to assess by either LM or CM.

Our findings suggest that the amount of myocilin and possibly its composition is altered in inherited POAG in the Beagle leading directly or indirectly to a gradual rise in the internal pressure of the eye. In order to determine this possibility further, we plan to examine specimens of individuals at different ages including those with no signs of the disease being exhibited, i.e., prior to rise in intraocular pressure. By understanding the factors responsible for the initial elevation of internal eye pressure during primary open-angle glaucoma, new methods to control the internal pressure may be developed, and most importantly, the knowledge collected from this research will allow us to reveal the underlying cause of this disease.

\section{References}

[1] Fingert JH, Stone EM, Sheffield VC, Alward WLM (2002) Myocilin Glaucoma. Survey of Ophthalmology. Vol. 47, No. 6, pp. 547-561.

[2] Polansky JR (2003) Current Perspectives on the TIGR/MYOC gene (Myocilin) and glaucoma. Ophthalmol. Clin. North Am. 16:515-527.

[3] Gelatt KN, Brooks DE, Samuelson DA (1998) Comparative Glaucomatology I: The Spontaneous Glaucomas. Journal of Glaucoma. 7:187-201.

[4] This study was supported by the Society for the Prevention of Blindness, the Jaqua Foundation, Gwathmey-Adams Laboratory for Vision Research and Alcon Pharmaceuticals.
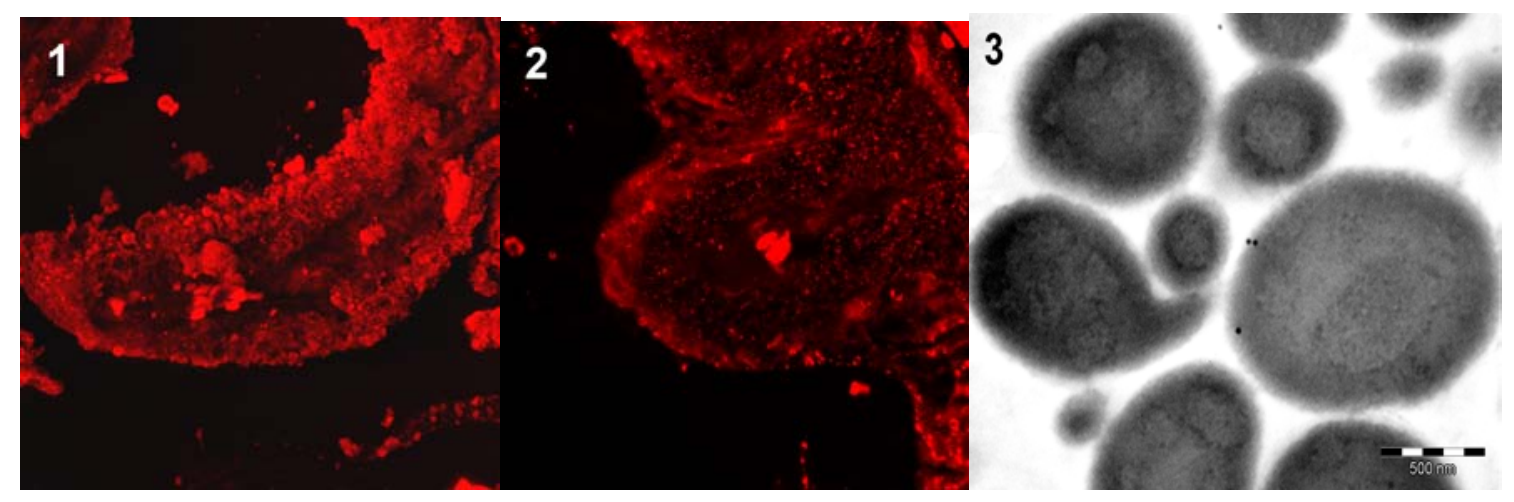

Fig. 1. Localization of myocilin by CM in the ciliary process of an adult Beagle with POAG. X1000. Fig. 2. Localization of myocilin by CM in the ciliary process of an age-matched adult Beagle. X1000. Fig. 3. Localization of myocilin by TEM in pigmented epithelium of the ciliary process of an adult Beagle with POAG. 